\title{
Clinical effect of conbercept on improving diabetic macular ischemia by OCT angiography
}

\author{
Ziyi Zhu', Youling Liang ${ }^{1}$, Bin Yan', Zhishang Meng ${ }^{1}$, Kejun Long', Yiwei Zhang ${ }^{1}$ and Jing Luo ${ }^{1,2^{*}}$ (D
}

\begin{abstract}
Background: Varying degrees of macular ischemia generally occur in diabetic retinopathy (DR). This study aims to evaluate the effect of conbercept with $3+$ pro re nata (PRN) on macular perfusion status in patients with diabetic macular edema (DME) and quantitatively assess changes in foveal avascular zone (FAZ) areas and capillary density in macular regions by applying optical coherence tomography angiography (OCTA).

Methods: Fifty patients were divided into ischemic $(n=31)$ and non-ischemic $(n=19)$ groups according to the presence of ischemia on OCTA at baseline. All patients received intravitreal injections of $0.5 \mathrm{mg}$ of conbercept with $3+$ PRN principle. The FAZ areas and macular vessel density measured using OCTA were evaluated at baseline, 3 months, and 6 months after treatment in both groups.

Results: At months 3 and 6 , the FAZ area in the ischemic group changed from $0.510 \pm 0.171 \mathrm{~mm}^{2}$ to $0.441 \pm 0.158 \mathrm{~mm}^{2}$ then to $0.427 \pm 0.153 \mathrm{~mm}^{2}(p=0.003, p=0.296)$; in the non-ischemic group, it remained stable $(p=0.269, p=0.926)$. The superficial vessel density changed from $41.1 \pm 4.1$ to $42.5 \% \pm 4.7 \%$ then to $42.6 \% \pm 4.6 \%(p=0.043, p=0.812)$, and the deep vessel density changed from $40.7 \pm 4.4$ to $42.3 \% \pm 3.6 \%$ then to $42.3 \% \pm 4.7 \%(p=0.072, p=0.961)$ in the ischemic group. In the non-ischemic group, the superficial vessel density changed from $44.8 \pm 3.2$ to $46.0 \% \pm 3.5 \%$ then to $45.7 \% \pm 3.3 \%$ ( $p=$ $0.108, p=0.666)$, whereas the deep vessel density changed from $43.6 \pm 3.6$ to $43.8 \% \pm 3.2 \%$ then to $43.5 \% \pm 4.5 \%$ ( $p=0.882$, $p=0.736)$. Reperfusion in macular nonperfusion areas was observed.
\end{abstract}

Conclusion: Anti-vascular endothelial growth factor treatment may have a positive effect on macular perfusion status. Furthermore, OCTA had advantages in quantifying and calculating blood flow index in the study of macular perfusion status.

Keywords: Diabetic retinopathy, Macular ischemia, Conbercept, OCT angiography

\section{Background}

As extensively reported, the further the diabetic retinopathy (DR) progresses, the severer the condition of diabetic macular ischemia (DMI) in patients with DR $[1,2]$. In macular ischemia, the structure of foveal capillary network is damaged. Such damage includes enlargement

\footnotetext{
* Correspondence: luojing001@csu.edu.cn

'Department of Ophthalmology, The Second Xiangya Hospital, Central South University, 139 Middle Renmin Rd, Changsha, Hunan 410011, People's Republic of China

${ }^{2}$ Hunan Clinical Research Center of Ophthalmic Disease, Changsha, Hunan 410011, People's Republic of China
}

and irregularity of the foveal avascular zone (FAZ) and appearance of macular nonperfusion (MNP), leading to macular function disturbance. According to ETDRS Report No. 11, the macular ischemia is defined as a FAZ area enlarging more than $1000 \mu \mathrm{m}$ in greatest diameter (supposing the FAZ is round or oval), equaling to the size of FAZ more than the area within $500 \mu \mathrm{m}$ radius circle, and/or broken perifoveal capillary rings at the borders of FAZ with areas of macular capillary nonperfusion within the al disk diameter of the foveal center according to fluorescein angiography (FA) [3]. In healthy

(c) The Author(s). 2020 Open Access This article is licensed under a Creative Commons Attribution 4.0 International License, which permits use, sharing, adaptation, distribution and reproduction in any medium or format, as long as you give appropriate credit to the original author(s) and the source, provide a link to the Creative Commons licence, and indicate if changes were made. The images or other third party material in this article are included in the article's Creative Commons licence, unless indicated otherwise in a credit line to the material. If material is not included in the article's Creative Commons licence and your intended use is not permitted by statutory regulation or exceeds the permitted use, you will need to obtain permission directly from the copyright holder. To view a copy of this licence, visit http://creativecommons.org/licenses/by/4.0/ The Creative Commons Public Domain Dedication waiver (http://creativecommons.org/publicdomain/zero/1.0/) applies to the data made available in this article, unless otherwise stated in a credit line to the data. 
people, the diameter of normal FAZ commonly varies from $500 \mu \mathrm{m}$ to $600 \mu \mathrm{m}$ (less than the area within $300 \mu \mathrm{m}$ radius circle equaling to $0.282 \mathrm{~mm}^{2}$ ) [3]. When a macular region is affected by ischemia, it presents in varying degrees, including disappearance of a part of the macular arch ring capillary network, expansion of the FAZ area, damage in perifoveal capillaries, and appearance of MNP area in the macular region. Different levels of macular ischemia can represent disease severity and progression; for instance, moderate-to-severe macular ischemia affects visual function seriously. Macular ischemia results from the occlusion of foveal capillary network, and vascular endothelial growth factor (VEGF) plays a vital role in the mechanism by which VEGF leads the closure of retinal vascular in patients with diabetic macular edema (DME) [4].

Several previous large studies regarding the treatment effects of anti-VEGF on DME, including DRCR studies, did not include macular ischemia as an observation indicator. To date, the insight into whether anti-VEGF therapy could aggravate retinal ischemia remains controversial. In the past decade, some clinical studies suggested that blocking VEGF might be harmful to retinal vascular integrity, especially in patients with preexisting retinal ischemia; in these patients, anti-VEGF therapy aggravated retinal ischemia because retinal nonperfusion (RNP) areas enlarged after anti-VEGF therapy $[5,6]$. However, increasing studies have shown that retinal ischemia does not worsen after anti-VEFG therapy but has no retinal reperfusion in the preexisted RNP areas [7-9]. After anti-VEGF treatment, the proportion of patients with $\geq 2$-step DR severity score improvement is greater in patients with MNP at baseline [10]. Meanwhile, few studies indicated that anti-VEGF therapy could reduce RNP progression. In a multicenter clinical trial, monthly injection of 0.3 or $0.5 \mathrm{mg}$ of ranibizumab can slow retinal vessel closure in DME and can be associated with retinal reperfusion at the anterior nonperfusion area in some patients [11]. Long-term anti-VEGF therapy to patients with DME could improve DR severity and even prevent worsening, especially in mild and severe DR cases. Furthermore, early treatment may prevent visual damage caused by proliferative DR (PDR) and obtain better therapeutic effects [12]. Thus, parts of researchers think anti-VEGF therapy not only improves patients' vision but also reduces RNP progression, and even enhances blood flow [11-13].

To date, most of the studies about anti-VEGF effects on DMI were small cross-sectional studies or retrospective studies, and they lack supporting prospective research. Moreover, most studies often use FA to observe macular ischemia changes; however, this tool is invasive and poorly repeatable. Meanwhile, optical coherence tomography angiography (OCTA) is noninvasive, repeatable, and easily operable, and it is subtler than FA in displaying macular area capillaries. OCTA acquires high- resolution images of macular areas by capturing the signals of moving red blood cells and provides images of the construction of macular capillaries at different plexus of the retina. In addition, OCTA is highly sensitive and consistent compared with FA [14-16]. However, numerous studies only focus on the retinal ischemia status, and more detailed effects on macular ischemia after anti-VEGF therapy have not been studied clearly. Thus, we decided to adopt OCTA as the main evaluation method to observe the blood flow of macular areas and quantify the density of macular vessels.

In this prospective study, we mainly aim to investigate the effects of conbercept with $3+$ PRN on macular perfusion status in patients with DME and to quantify FAZ areas and capillary density in the macular region. Conbercept (Langmu; Kanghong, Inc., Sichuan, China) is a new drug comprising a VEGF receptor (VEGFR) fusion protein, with a high binding affinity to VEGF and a long half-life in vitreous, and it has been proven to reduce the chances of intraoperative bleeding in vitrectomy procedures by decreasing VEGF concentrations. Conbercept demonstrates a higher binding activity with VEGF-A, VEGF-B, and placental growth factor (PIGF) than ranibizumab. Clinical trials indicate that intravitreal injections of conbercept can improve visual acuity in patients receiving vitrectomy and seems to reduce the recurrence rate of vitreous hemorrhage in patients with PDR [17].

\section{Methods}

The study followed the tenets of the Declaration of Helsinki, all patients gave informed consent before participation in this study and all procedures performed were in accordance with the ethical standards of the Second Xiangya Hospital of Central South University committee. In this study, 50 patients (obtained after informed consent), who consulted in the abovementioned hospital for diminution of vision caused by diabetes between September 2018 and March 2019, were diagnosed of DR with different levels of DME [18]. And only one eye of each patient was included in the study. Inclusion criteria were as follows: patients $>18$ years old with type 1 or 2 diabetes; and definite retinal thickening due to DME as the main cause of visual loss (central fovea thickness $[\mathrm{CFT}] \geq 250 \mu \mathrm{m}$ measured on OCT). Meanwhile, the exclusion criteria were the following: visual loss caused by any other retinal disease, excluding DME; retinal treatment or major ocular surgery within the prior 6 months; intraocular pressure $\geq 25 \mathrm{mmHg}$; refractive error (myopia $>6 \mathrm{D}$ or hyperopia $>3 \mathrm{D}$ ), severe refractive media, and other systemic diseases that needed hospitalization. All of them were injected with $0.5 \mathrm{mg}$ of conbercept at monthly intervals for the first 3 months and given additional interval injections according to the pro re nata (PRN) principle [19]. All of these patients 
were evaluated for 6 months, comprising a 3-month treatment period (received monthly interval injection of $0.5 \mathrm{mg}$ of conbercept) and a 3-month observation period (received treatment according to the PRN principle). Before the first intraocular injection, all patients underwent FA, OCT, and OCTA, which showed whether a patient had apparent macular ischemia (including FAZ area expansion, perifoveal capillary ring damage, and/or MNP appearance). And we excluded patients with poor OCTA image quality before grouping patients: Q-score below 7; opacity of refractive media; presence of significant residual motion artifacts; anatomical features of macular area severely disrupted (such as severe cystoid macular edema) leading to segmentation errors. Then, the patients were divided into two groups, namely, the ischemic group and the non-ischemic group. Considering the lack of uniform definition of macular ischemia classification according OCTA, we adopted the basis of grouping at baseline according to macular ischemic grading defined by FA [3]. Ischemic group is defined as the presence of one or more of the following criteria: 1.FAZ area is greater than or equal to $1000 \mu \mathrm{m}$ diameter circle $(0.785 \mathrm{~mm} 2) ; 2$. Outline of FAZ is not a smoothly regular round or oval, the capillary outline is lost or destroyed; 3. Appearance of retinal capillary loss even MNPs in macular region. The rest is defined as nonischemic group.

\section{Image acquisition}

All OCT and OCT angiography images were acquired from AngioVue OCT system version 2018.0.0.14 (RTVue XR Avanti, Optovue Inc., Fremont, CA, USA.) using splitspectrum amplitude decorrelation angiography (SSADA) algorithm to detect blood flow and provide a new for rapid imaging of detailed microvasculature at distinct depths [15]. The system reduced artifacts by motion correction technology (MCT) and 3D projection artifact removal (PAR) remove artifacts, and PAR differentiated situ OCTA signal from projection artifact and removed the projection artifacts $[20,21]$. The scanning area of OCTA was uniformly obtained in $6 \times 6 \mathrm{~mm}^{2}$ sections consisting of 304 B-scans. Every B-scan was repeated twice, as well as 304 A-scans. In 3 s, 209000 A-scans were obtained. AngioVue provided an automated software algorithm to generate the boundaries of superficial capillary plexus (from ILM to $10 \mu \mathrm{m}$ above IPL), deep capillary plexus (from $10 \mu \mathrm{m}$ above IPL to $10 \mu \mathrm{m}$ below OPL) and choriocapillaris. And the boundary of FAZ area was from ILM to $10 \mu \mathrm{m}$ below OPL. While the segmentation boundaries also could be adjusted and corrected by manually segmented [22].

\section{Data measurement}

We assessed best corrected visual acuity (BCVA) by using a tumbling $\mathrm{E}$ chart at an initial testing distance of
$5 \mathrm{~m}$, and the result was displayed in a $\log M A R$ unit format [23]. Then, we calculated the CFT as an average value within a circular $1 \mathrm{~mm}$ diameter area centered in the fovea measured by OCT. We also obtained vessel density value by calculating the percentage of blood vessel area in a $6 \times 6 \mathrm{~mm}^{2}$ selected area from the OCTA. Furthermore, FAZ area was calculated using an automated software but could be corrected by manual selection.

\section{Statistical analysis}

Main measurements, including BCVA, FAZ area, CFT, and superficial and deep vessel density, in this study were tested by Kolmogorov-Smirnov test $(p>0.05$ in all samples). Hence, we adopted $t$ test principally. At months 0,3 , and 6, changes in Q-score, CFT, FAZ area, and blood flow were evaluated by paired-sample $t$ test. Moreover, $p<0.05$ indicated statistical significance. Statistical analysis was performed using the SPSS 25.0 (SPSS Inc., Chicago, III, USA).

\section{Results}

\section{Characteristics of patients with DME at baseline}

This study enrolled 50 patients with DME and divided them into two groups, namely, the ischemic group $(n=$ $31)$ and the non-ischemic group $(n=19)$. The characteristics of patients at baseline are shown in Table 1. These patients comprised 24 males and 26 females, and the mean age was 55.8 years (standard deviation [SD]: 8.4 years [3480 years]). The BCVA was $0.61 \pm 0.34($ mean $\pm S D)$. The mean duration of diabetes was 9.4 years, and the HbA1c was $9.6 \% \pm 2.0 \%$. Among these 50 patients, 7 had moderate NPDR (14\%), 34 had severe NPDR (68\%), and 9 had PDR (18\%). Among the 9 patients with DR, 9 had hypertension, 4 had nephropathy, and 1 had thrombocytopenia, showing that DR is associated with many other systemic disorders, especially circulatory diseases and nephropathy. The overall main characteristics of patients with DME were increased central fovea thickness $(367 \pm 122 \mu \mathrm{m})$, increased FAZ area $\left(0.455 \pm 0.171 \mathrm{~mm}^{2}\right)$, and deceased vessel density (vessel density in superficial plexus [ILM-OPL] and deep plexus (IPL-OPL) was $42.5 \pm 4.2$ and $41.8 \% \pm$ $4.3 \%$, respectively).

The composition of sex and age, BCVA, diabetes duration, and $\mathrm{GHb}$ between the ischemic group and nonischemic group had no significant difference $(p>0.05$, chi-square test and independent-sample $t$ test). Conversely, the CFT, FAZ area, and vessel density between the two groups were significantly different. In the ischemic group, the CFT $(328 \pm 109 \mu \mathrm{m})$ was significantly lower than that in the non-ischemic group $(p=0.005)$. The FAZ area in the ischemic group $(0.510 \pm 0.171$ $\mathrm{mm}^{2}$ ) was preoperatively larger than that in the ischemic group $\left(0.364 \pm 0.127 \mathrm{~mm}^{2}, p=0.001\right)$. Additionally, the 
Table 1 Basic characteristics of patients at baseline $(n=50)$

\begin{tabular}{|c|c|c|c|}
\hline & Ischemic Group ( $n=31$ ) & Non-ischemic Group $(n=19)$ & Total $(n=50)$ \\
\hline \multicolumn{4}{|l|}{ Gender, n } \\
\hline Male & $17(55 \%)$ & $7(49 \%)$ & $24(48 \%)$ \\
\hline Female & $14(45 \%)$ & $12(51 \%)$ & $26(52 \%)$ \\
\hline Age, years & $55.8 \pm 8.5$ & $55.8 \pm 8.4$ & $55.8 \pm 8.4$ \\
\hline BCVA, logMAR units & $0.64 \pm 0.34$ & $0.56 \pm 0.34$ & $0.61 \pm 0.34$ \\
\hline Duration of Diabetes, years & $10.1 \pm 4.5$ & $8.2 \pm 4.9$ & $9.4 \pm 4.7$ \\
\hline \multicolumn{4}{|l|}{ Stage of DR, n } \\
\hline Mild NPDR & $0(0)$ & $0(0)$ & $0(0)$ \\
\hline Moderate NPDR & $4(13 \%)$ & $3(16 \%)$ & $7(14 \%)$ \\
\hline Severe NPDR & $21(68 \%)$ & $13(68 \%)$ & $34(68 \%)$ \\
\hline PDR & $6(19 \%)$ & $3(16 \%)$ & $9(18 \%)$ \\
\hline $\mathrm{GHb}, \%$ & $9.6 \pm 2.1$ & $9.7 \pm 2.0$ & $9.6 \pm 2.0$ \\
\hline \multicolumn{4}{|l|}{ Complication } \\
\hline Hypertension & 7 & 2 & 9 \\
\hline Renal Failure & 2 & 2 & 4 \\
\hline Others & 0 & 1 & 1 \\
\hline CFT(1 mm), $\mu \mathrm{m}$ & $328 \pm 109$ & $430 \pm 118$ & $367 \pm 122$ \\
\hline FAZ area, mm2 & $0.510 \pm 0.171$ & $0.364 \pm 0.127$ & $0.455 \pm 0.171$ \\
\hline \multicolumn{4}{|l|}{ Vessel Density,\% } \\
\hline Superficial (ILM-IPL) & $41.1 \pm 4.1$ & $44.8 \pm 3.2$ & $42.5 \pm 4.2$ \\
\hline Deep (IPL-OPL) & $40.7 \pm 4.4$ & $44.8 \pm 3.2$ & $41.8 \pm 4.3$ \\
\hline
\end{tabular}

NPDR non-proliferative diabetic retinopathy, PDR proliferative diabetic retinopathy, GHb glycated hemoglobin

superficial and deep vessel densities in the ischemic groups were $41.1 \pm 4.1$ and $40.7 \% \pm 4.4 \%$, respectively; both were clearly low than those in the non-ischemic group $(44.8 \% \pm 3.2 \%, p=0.001$ and $44.8 \pm 3.2 \%, p=$ 0.015). Overall, the patients in the ischemic group showed ischemic changes such as FAZ area expansion, MNP appearance, and blood flow density decrement.

\section{Anti-VEGF therapy decreased the FAZ area}

All patients were intraocularly injected with $0.5 \mathrm{mg}$ of conbercept according to the $3+$ PRN principle. The main observation point was set up at months 0,3 , and 6 . Additionally, we analyzed comparing the Q-score (pairedsample $t$ test) for each time point to estimate whether Q-score affected the results in our study. There had no significant difference of Q-score among different time points. In the ischemic group, Q-score was $7.74 \pm 0.82$ at baseline, $7.90 \pm 1.03(p=0.476)$ at month 3 and $8.07 \pm$ $0.92(p=0.587)$ at month 6 . And in the non-ischemic group, Q-score was $7.79 \pm 0.79$ at baseline, $7.74 \pm 0.65$ $(p=0.790)$ at month 3 and $8.00 \pm 1.00(p=0.350)$ at month 6 . The changes in the FAZ area in both groups are depicted in Fig. 1.

After the patients were intraocularly injected with 0.5 $\mathrm{mg}$ of conbercept thrice, their BCVA improved and CFT decreased in both groups. The FAZ area of all patients decreased significantly at month $3(p=0.017)$, and remained stable until the month $6(p=0.311)$. Especially in the ischemic group, the FAZ area decreased from $0.510 \mathrm{~mm}^{2}$ to $0.441 \mathrm{~mm}^{2}(p=0.003)$, continually decreasing to $0.427 \mathrm{~mm}^{2}$ at the end point $(p=0.296)$. In contrast, the FAZ area did not expand significantly in the non-ischemic group. A representative case of DMI showed an apparent decrease in FAZ area, as illustrated in Fig. 2.

\section{Anti-VEFG therapy improved the vessel density in the macular region}

The superficial vessel density of all patients increased significantly at month 3 ( $\left.\mathrm{p}_{\text {month3 }}=0.008\right)$, and remained stable until the month 6 ( $\left.\mathrm{p}_{\text {month6 }}=0.944\right)$. The changes in the vessel density in both groups are depicted in Fig. 3. In the ischemic group, the superficial vessel density increased from $41.1 \% \pm 4.1 \%$ to $42.5 \% \pm 4.7 \%$ then to $42.6 \pm 4.6 \%$ at the end point, and the deep vessel density changed from $40.7 \% \pm 4.4 \%$ to $42.3 \pm 3.6 \%$ then to $42.3 \% \pm 4.7 \%$ at month 6 . The capillary density increased in both superficial plexus ( $\mathrm{p}_{\text {month3 }}=0.043$ ) and deep plexus $\left(\mathrm{p}_{\text {month3 }}=0.072\right.$ ) in the first 3 months after antiVEGF therapy, especially in the superficial plexus. 


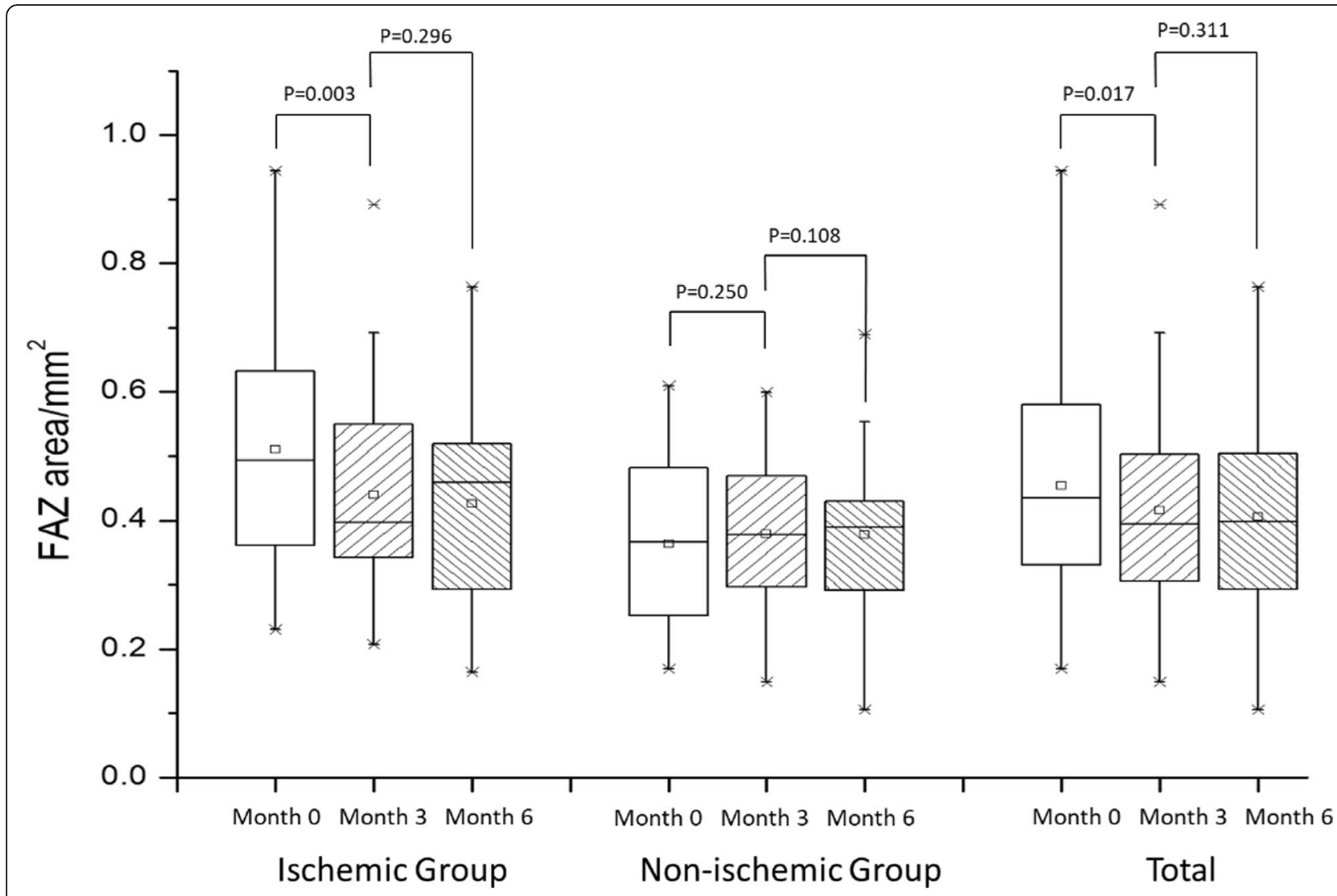

Fig. 1 Changes in FAZ area after anti-VEFG therapy

Moreover, the vessel density remained stable during the observation period $\left(p_{\text {month }}=0.812, p_{\text {month6 }}=0.961\right)$. In the non-ischemic group, the superficial vessel density changed from $44.8 \% \pm 3.2 \%$ to $46.0 \% \pm 3.5 \%$ then to $45.7 \pm 3.3 \%$ ( pmonth3 $=0.108$, p ponth6 $=0.666$ ), whereas the deep vessel density changed from $43.6 \pm 3.6$ to $43.8 \% \pm 3.2 \%$ then to $43.5 \pm 4.5 \%$ at the end point ( $\left.\mathrm{p}_{\text {month3 }}=0.882, \mathrm{p}_{\text {month6 }}=0.736\right)$. Some patients had increased vessel density and reperfusion in previous nonperfusion area in the macular region (Fig. 4).

\section{Discussion}

In our study, varying degrees of macular ischemia gained different therapeutic effects after anti-VEGF therapy in both the ischemic and non-ischemic groups. Furthermore, the macular perfusion status improved after the anti-VEGF therapy, especially in the FAZ area and superficial vessel density. The superficial vessel density is associated with DME development, whereas the vessel density in deep plexus corresponds to macular photoreceptors and is important to the oxygen requirements of photoreceptors and outer retina in patients with DMI. Some study found that increased number of capillary drop-out in superficial capillary plexus correlated with DR severity [24, 25]. Some patients with DMI obtained reperfusion at previous nonperfusion areas. As observed in Fig. 4, the OCTA detected that the FAZ area of a 59year-old patient with DME, the structure of arch ring capillaries already disrupted, clearly decreased after receiving the anti-VEGF therapy and indicating rebuilding especially in the inferior-nasal macular fovea. Therefore, the anti-VEGF therapy improved macular ischemia and blood supply even the occurrence of macular reperfusion in some patients with DME.

Macular ischemia is a contraindication of anti-VEFG therapy, but some patients developed capillary nonperfusion after such therapy. Severe macular ischemia may be a limitation of visual acuity outcomes in patients with DME after receiving anti-VEGF therapy [26]. However, trials in DME suggested that anti-VEGF therapy did not induce retinal ischemia at least in healthy retina. In addition, repeated anti-VEGF therapy on macular perfusion in patients with DME did not cause treatmentrelated significant changes in FAZ sizes and capillary loss around the fovea. Patients with DME who had severe ischemia still achieved favorable changes in BCVA and central macular thickness after long-term antiVEGF therapy [6, 7]; thus, anti-VEGF therapy may be an alternative for such patients. Therefore, patients with severe macular ischemia receiving anti-VEFG therapy 

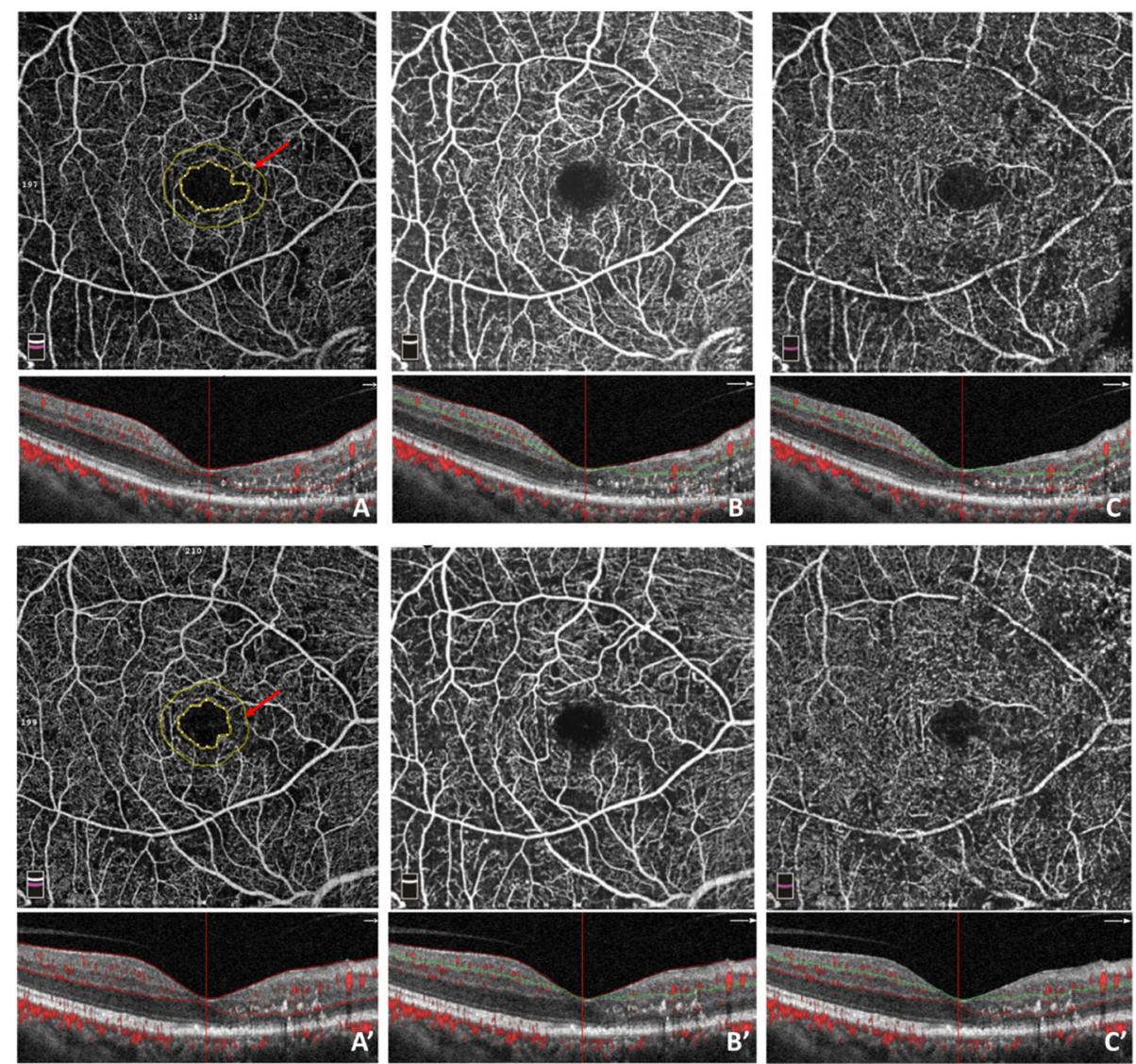

Fig. 2 FAZ area clearly decreasing in the eye of a 59-year-old male, 6 months after receiving conbercept treatment. Notes: OCT angiography images (a-c) depict macular ischemia and apparent FAZ expansion captured in $6 \times 6 \mathrm{~mm} 2$ sections in the macular area at month 0, Q-score $=8 /$ 10. a FAZ area with $0.604 \mathrm{~mm} 2$, showing severe FAZ area expansion and arch ring capillary network breakage; b Superficial capillary plexus (ILMIPL), vessel density of 39.2\%; c Deep capillary plexus (IPL-OPL), vessel density of 43.5\%; At month 6, OCT angiography images (a'-c' $\left.\mathbf{c}^{\prime}\right)$ showed FAZ area decrease, Q-score $=9 / 10$. a' FAZ area with $0.464 \mathrm{~mm}$; b Superficial capillary plexus vessel density of 40.4\%; c Deep capillary plexus vessel density of $43.1 \%$
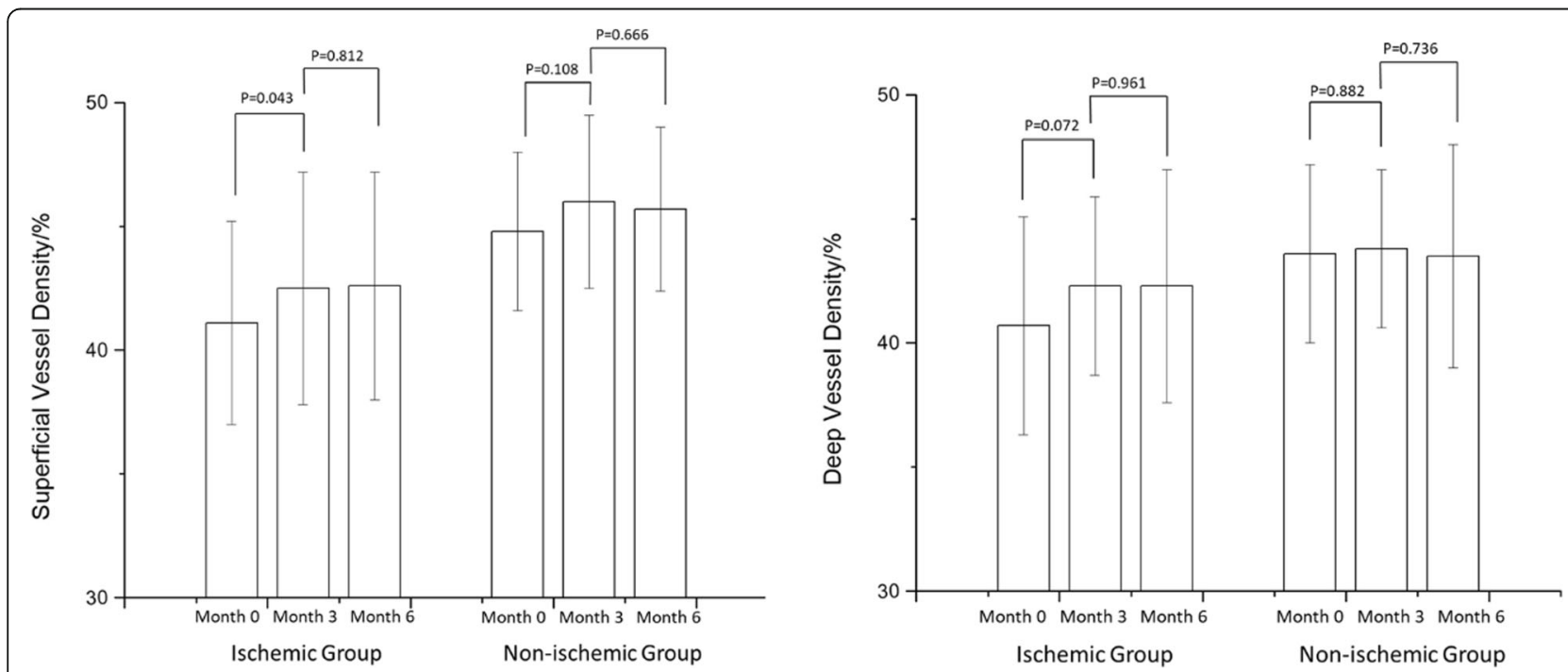

Fig. 3 Changes of vessel density in both superficial plexus and deep plexus in all patients 

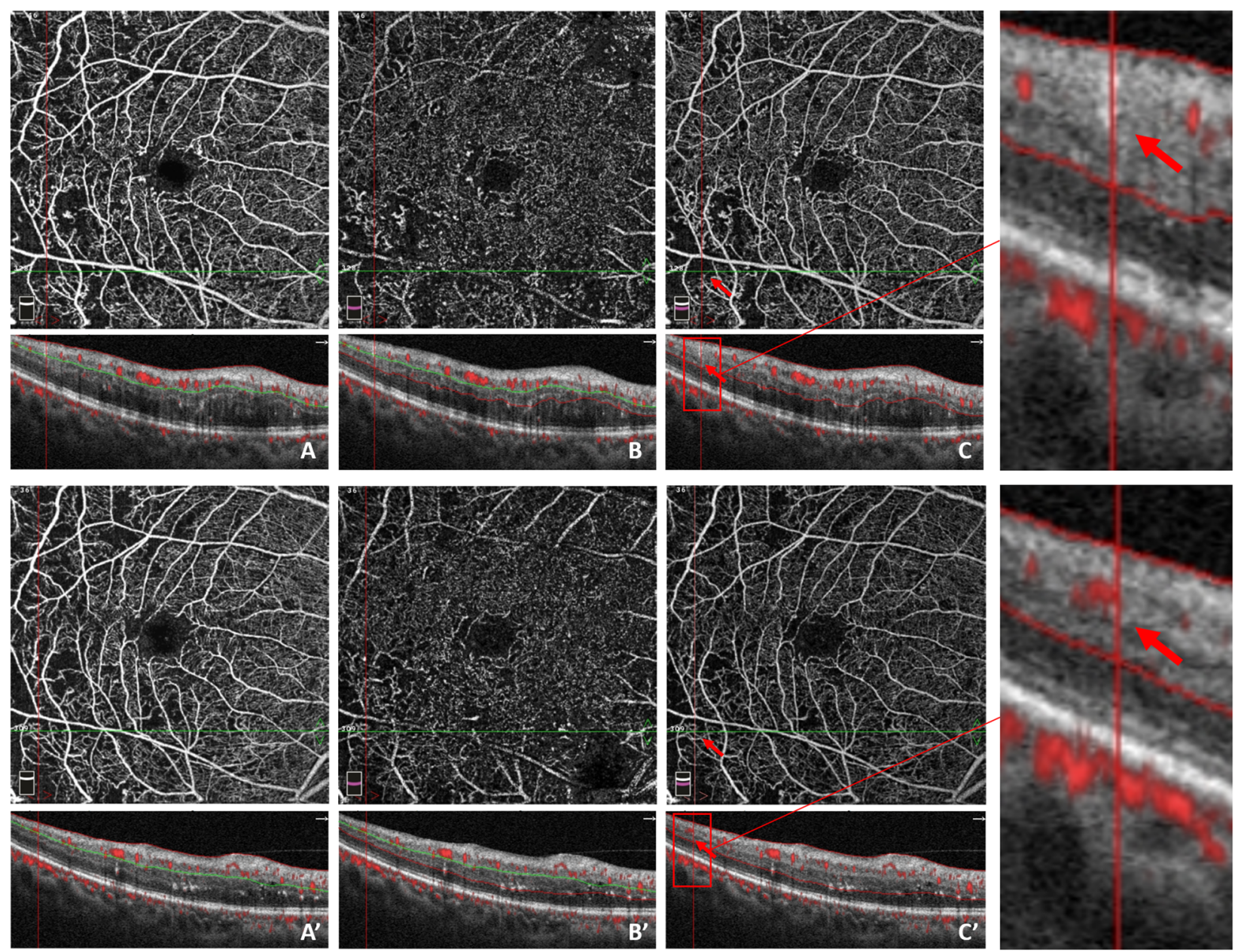

Fig. 4 Reperfusion occurred in the right eye of a 53-year-old male, 6 months after receiving conbercept treatment. Red arrows and boxes indicate growth of new blood flow signal and reperfusion in the macular nonperfusion areas. OCT angiography images (a-c) at baseline illustrate the macular nonperfusion area, Q-score =8/10. a Superficial capillary plexus, VD =45.7\%; b Deep capillary plexus, VD = 39.6; c Full retinal capillary plexus. OCT angiography images $\mathbf{a}^{\prime}-\mathbf{c}^{\prime}$ at month 6 indicate reperfusion in previous macular nonperfusion areas, Q-score $=8 / 10$. $\mathbf{a}$ Superficial capillary plexus, VD $=45.5 \%$; $\mathbf{b}$ Deep capillary plexus, VD $=40.2 \%$; c Full retinal capillary plexus

should be individualized, and they need a comprehensive assessment of possible risks and closer follow-up to prevent the worsening of ischemia. Overall, patients with DMI in our study experienced improved eyesight and macular ischemia after anti-VEGF therapy.

Highly vitreous concentration of VEGF can lead to serious retina ischemia and hypoxia [27]. The retinal arterioles would contract immediately, and once ischemia occurs, vascular occlusion and RNP areas progression take place, resulting in retinal microvascular abnormality and neovascularization elsewhere, especially in patients with severe NPDR and PDR [28]. VEGF is the strongest angiogenic factor. The VEGF family in mammals mainly includes five species and PlGF. In DR, VEGF and PlGF disrupt the blood-retinal barrier, leading to DMI progression. Anti-VEFG therapy improves retinal hypoxia and ischemia by reducing the VEGF content.
Conbercept is a new VEGFR fusion protein that reduces the concentration of VEGF and PIGF by specifically binding to VEGF-A, VEGF-B, and PlGF [29]. However, the molecular mechanism on how anti-VEGF therapy improves ischemia and realizes reperfusion remains unclear. A study using retinal ischemia animal models proved that anti-VEGF therapy could reduce autophagy and apoptosis rate and activate ischemia-damaged microglia to protect the retinal ganglion cells and bipolar cells [30]. In another study on tumor, VEGF inhibition can normalize peripheral cells, stabilize the basement membrane, remodel the immature vessels to a more mature version by destroying the vessels that lack peripheral cells, and provide frameworks for new vessels to grow in again by stabilizing the basement membrane. Surmising that the mechanism is the same in the retina $[31,32]$. 
In this study, we adopted OCTA as the main evaluation method to observe the blood flow of the macular region and to quantify the macular vessel density. Despite acknowledging FA as a gold standard in the diagnosis of DR and classification of DMI, by comparing the FA and OCTA, we found that both tests were consistent in showing the vessels in the macular area, but OCTA is unacted on the leakage of fluorescein [14-16]. OCTA not only evaluated macular ischemia and DR severity but also predicted the peripheral nonperfusion by observing the change of FAZ size [33]. However, OCTA still has several defects. Fluid may induce segmentation artifacts, and OCTA quantitative metrics lacks consensus and normative database. And the main problem is that projection artifacts from large superficial vessels were inevitably included in the OCTA images of deep plexus, although we used PAR algorithm to reduce decorrelation tailing and projection artifact. This is a big challenge for us to measure the truly values of deep vessel density. But we believe that with the development of artifact removal algorithms, the measurements in vessel density might more precisely represent the actual flow especially in deep plexus. Other limitations in our study mainly include the small sample size and the short study period. Indeed, a part of studies used OCTA observing changes in macular perfusion and found there is no improvement in FAZ and vessel density after anti-VEGF [34-37]. The effects of anti-VEGF therapy on DMI need a larger sample size and a longer observation period to determine how anti-VEGF plays a role in improving macular ischemia and retinal ischemia and realizing reperfusion in nonperfusion areas.

\section{Conclusions}

In our study, we observed the improvements of FAZ area and superficial vessel density in patients with DME after receiving intraocular injection of conbercept according to the $3+$ PRN principle, especially greater in patients with DMI at baseline. Furthermore, conbercept had a positive impact on macular perfusion status and promoted reperfusion in macular nonperfusion areas. Lastly, OCTA may be used as an important imaging modality to evaluate retinal vasculature and to quantify the perfusion status in DR.

\section{Supplementary information}

Supplementary information accompanies this paper at https://doi.org/10. 1186/s12886-020-01648-x.

\section{Additional file 1:.}

\section{Abbreviations}

DR: Diabetic retinopathy; DMI: Diabetic macular ischemia; DME: Diabetic macular edema; FAZ: Foveal avascular zone; MNP: Macular nonperfusion; RNP: Retinal nonperfusion; VEGF: Vascular endothelial growth factor; VEGF
R: Vascular endothelial growth factor receptor; PIGF: Placental growth factor; FA: Fluorescein angiography; OCT: Optical coherence tomography; OCTA: Optical coherence tomography angiography; CFT: Central fovea thickness; PRN: Pro re nata; SSADA: Split-spectrum amplitude decorrelation angiography; MCT: Motion correction technology; PAR: Projection artifact removal; BCVA: Best corrected visual acuity; ILM: Internal limiting membrane; IPL: Inner plexiform layer; OPL: Outer plexiform layer; NPDR: Non-proliferative diabetic retinopathy; PDR: Proliferative diabetic retinopathy; GHb: Glycated hemoglobin

\section{Acknowledgements \\ Not applicable.}

\section{Authors' contributions}

$Z Y Z, Y L L$ and $J L$ were responsible for the conception and design of this review. ZYZ, BY, KJL and YWZ acquired the data. ZYZ and ZSM analyzed and interpreted the data. ZYZ wrote the draft. YLL and JL revised the manuscript critically. The manuscript did not benefit from the use of other editorial services. No any authors on earlier versions have been removed. All authors have read and approved the final manuscript.

Funding

Not applicable.

\section{Availability of data and materials}

All data is included in Excel sheet, and medical records and picture information is included in this published article and supplementary files.

\section{Ethics approval and consent to participate}

The study was approved by the Ethics Committee of the Second Xiangya Hospital of Central South University. All research was conducted in accordance with the Declaration of Helsinki, and all patients gave informed consent before participation in this study. Subjects were provided a written informed consent in accordance with the guidelines of the Second Xiangya Hospital of Central South University.

\section{Consent for publication}

Not Applicable.

\section{Competing interests}

The authors declare that they have no competing interests.

Received: 19 December 2019 Accepted: 17 September 2020

Published online: 25 September 2020

\section{References}

1. Arend $\mathrm{O}$, et al. Retinal microcirculation in patients with diabetes mellitus: dynamic and morphological analysis of perifoveal capillary network. Br J Ophthalmol. 1991;75(9):514-8..

2. Yalcin NG, Ozdek S. The relationship between macular cyst formation and ischemia in diabetic macular edema. Turk J Ophthalmol. 2019;49(4):194-200.

3. Early Treatment Diabetic Retinopathy Study Research Group. Classification of diabetic retinopathy from fluorescein angiograms: ETDRS report number 11. Ophthalmology. 1991;98(5 Suppl):807-22.

4. Usman M. An overview of our current understanding of diabetic macular ischemia (DMI). Cureus. 2018;10(7):e3064.

5. Li JK, et al. Changes in vitreous VEGF, bFGF and fibrosis in proliferative diabetic retinopathy after intravitreal bevacizumab. Int J Ophthalmol. 2015;8(6):1202-6.

6. Bonini-Filho $\mathrm{M}$, et al. Intravitreal bevacizumab for diabetic macular edema associated with severe capillary loss: one-year results of a pilot study. Am J Ophthalmol. 2009;147(6):1022-30 1030.e1-5.

7. Karst SG, et al. Association of Changes in macular perfusion with Ranibizumab treatment for diabetic macular edema: a subanalysis of the RESTORE (extension) study. JAMA Ophthalmol. 2018;136(4):315-21.

8. Bonnin S, et al. Anti-vascular endothelial growth factor therapy can improve diabetic retinopathy score without change in retinal perfusion. Retina. 2019; 39(3):426-34.

9. Couturier A, et al. Widefield OCT-angiography and fluorescein angiography assessments of nonperfusion in diabetic retinopathy and edema treated with anti-vascular endothelial growth factor. Ophthalmology. 2019;126(12):1685-94. 
10. Reddy RK, et al. Efficacy of ranibizumab in eyes with diabetic macular edema and macular nonperfusion in RIDE and RISE. Ophthalmology. 2018; 125(10):p1568-74.

11. Campochiaro PA, et al. Neutralization of vascular endothelial growth factor slows progression of retinal nonperfusion in patients with diabetic macular edema. Ophthalmology. 2014;121(9):1783-89.

12. Ip MS, et al. Long-term effects of therapy with ranibizumab on diabetic retinopathy severity and baseline risk factors for worsening retinopathy. Ophthalmology. 2015;122(2):367-74.

13. Ariana $\mathrm{L}$, Irene $\mathrm{R}$, Anton $\mathrm{O}$, et al. Retinal, reperfusion in diabetic retinopathy following treatment with anti-VEGF intravitreal injections [J]. Clin Ophthalmol. 2017;11:193-200.

14. Garcia JM, et al. Diabetic macular ischemia diagnosis: comparison between optical coherence tomography angiography and fluorescein angiography. J Ophthalmol. 2016;2016:3989310.

15. Jia $Y$, et al. Split-spectrum amplitude-decorrelation angiography with optical coherence tomography. Opt Express. 2012;20(4):4710-25.

16. Cole ED, et al. Contemporary retinal imaging techniques in diabetic retinopathy: a review. Clin Exp Ophthalmol. 2016:44(4):289-99.

17. Ren $X$, et al. Safety and efficacy of intravitreal conbercept injection after vitrectomy for the treatment of proliferative diabetic retinopathy. Eye (Lond). 2019;33(7):1177-83.

18. Early Treatment Diabetic Retinopathy Study research group. Photocoagulation for diabetic macular edema: ETDRS report number 1. Arch Ophthalmol. 1985;103(12):1796-806.

19. Larsen M, et al. Individualized Ranibizumab regimen driven by stabilization criteria for central retinal vein occlusion: twelve-month results of the CRYS TAL study. Ophthalmology. 2016;123(5):1101-11.

20. Camino A, et al. Evaluation of artifact reduction in optical coherence tomography angiography with real-time tracking and motion correction technology. Biomed Opt Express. 2016;7(10):3905-15.

21. Hwang TS, Zhang M, Bhavsar K, et al. Visualization of 3 distinct retinal plexuses by projection-resolved optical coherence tomography angiography in diabetic retinopathy. JAMA Ophthalmol. 2016;134(12):1411-9.

22. Lavia C, et al. Vessel density of superficial, intermediate, and DEEP capillary plexuses using optical coherence tomography angiography. Retina. 2019; 39(2):247-58.

23. Ferris FR, et al. New visual acuity charts for clinical research. Am J Ophthalmol. 1982;94(1):91-6.

24. Schottenhamml J, et al. An automatic, intercapillary area-based algorithm for quantifying diabetes-related capillary dropout using optical coherence tomography angiography. Retina. 2016;36(Suppl 1):S93-S101.

25. Krawitz BD, et al. Parafoveal nonperfusion analysis in diabetic retinopathy using optical coherence tomography angiography. Transl Vis Sci Technol. 2018;7(4):4.

26. Douvali $\mathrm{M}$, et al. Effect of macular ischemia on intravitreal ranibizumab treatment for diabetic macular edema. Ophthalmologica. 2014;232(3):136-43.

27. Liu Y, et al. Reversible retinal vessel closure from VEGF-induced leukocyte plugging. JCl Insight. 2017:2(18).

28. El DY, et al. Reduced baseline diameter and contraction of peripheral retinal arterioles immediately after remote ischemia in diabetic patients. Graefes Arch Clin Exp Ophthalmol. 2019;257(10):2095-101.

29. Zhou J, et al. Concentrations of VEGF and PIGF decrease in eyes after Intravitreal Conbercept injection. Diabetes Ther. 2018;9(6):2393-8.

30. Palmhof $M$, et al. Fewer functional deficits and reduced cell death after Ranibizumab treatment in a retinal ischemia model. Int J Mol Sci. 2018;19(6):1636.

31. Inai T, et al. Inhibition of vascular endothelial growth factor (VEGF) signaling in cancer causes loss of endothelial fenestrations, regression of tumor vessels, and appearance of basement membrane ghosts. Am J Pathol. 2004;165(1):35-52.

32. Dickson PV, et al. Bevacizumab-induced transient remodeling of the vasculature in neuroblastoma xenografts results in improved delivery and efficacy of systemically administered chemotherapy. Clin Cancer Res. 2007; 13(13):3942-50.

33. Sim DA, et al. Patterns of peripheral retinal and central macula ischemia in diabetic retinopathy as evaluated by ultra-widefield fluorescein angiography. Am J Ophthalmol. 2014;158(1):144-53 e1.

34. Falavarjani $\mathrm{KG}$, et al. Optical coherence tomography angiography analysis of the foveal avascular zone and macular vessel density after anti-VEGF therapy in eyes with diabetic macular edema and retinal vein occlusion. Invest Ophthalmol Vis Sci. 2017:58(1):30-4
35. Dastiridou A, et al. OCT Angiography Study of the Macula in Patients with Diabetic Macular Edema Treated with Intravitreal Aflibercept [published online ahead of print, 2020 Jan 17]. Ocul Immunol Inflamm. 2020;1-6. https://doi.org/10.1080/09273948.2019.1704028.

36. Sorour OA, et al. Optical coherence tomography angiography analysis of macular vessel density before and after anti-VEGF therapy in eyes with diabetic retinopathy. Int Ophthalmol. 2019;39(10):2361-71.

37. Conti FF, et al. Changes in retinal and choriocapillaris density in diabetic patients receiving anti-vascular endothelial growth factor treatment using optical coherence tomography angiography. Int J Retina Vitreous. 2019;5:41.

\section{Publisher's Note}

Springer Nature remains neutral with regard to jurisdictional claims in published maps and institutional affiliations.
Ready to submit your research? Choose BMC and benefit from:

- fast, convenient online submission

- thorough peer review by experienced researchers in your field

- rapid publication on acceptance

- support for research data, including large and complex data types

- gold Open Access which fosters wider collaboration and increased citations

- maximum visibility for your research: over $100 \mathrm{M}$ website views per year

At BMC, research is always in progress.

Learn more biomedcentral.com/submissions 